denn Richtlinien bestimmen.“ (S. 19) Der von Sebastian Bröchler und Julia von Blumenthal herausgegebene Sammelband „Regierungskanzleien im politischen Prozess“ behandelt somit ein zentrales, jedoch bei weitem nicht hinreichend erforschtes Instrument politischer Führung. Basierend auf insgesamt neun Beiträgen einer Tagung der DVPW-Sektion „Regierungssystem und Regieren in der Bundesrepublik Deutschland“ aus dem Jahr 2007 leistet der Band dreierlei: Erstens bietet er einen Überblick über Stand und Perspektiven der Regierungszentralenforschung im internationalen Vergleich. Einen zweiten Schwerpunkt bildet die Auseinandersetzung mit den Funktionen und der Arbeitsweise des Bundeskanzleramts. Drittens wird auf die Rolle von Staatskanzleien im Politikmanagement von Landesregierungen, hier vor allem in Nordrhein-Westfalen, eingegangen.

Friedbert Rüb geht in seinem Beitrag davon aus, dass die Bedeutung der Kernexekutiven im politischen Prozess zugenommen hat. Gleichzeitig distanziert er sich vom „Regierungsstilimpressionismus“ (S. 78) und schlägt stattdessen eine Regierungsstiltypologie vor. An den Kanzlerschaften von Kohl, Schröder und Merkel wird exemplarisch geprüft, wie deren Regierungsstile die Arbeitsweise des Bundeskanzleramtes beeinflussten. Einen anderen Weg wählt Julia Fleischer, indem sie die Rolle des Bundeskanzleramtes in der exekutiven Entscheidungsfindung aus der Perspektive der neo-institutionalistischen Organisationstheorie beleuchtet. Während eine „wachsende Dominanz des Ressortprinzips und eine zunehmende funktionale Politisierung der Ministerialverwaltung “ (S. 140) festzustellen seien, beeinflussten Organisationsveränderungen die Steuerungsfähigkeit der Regierungszentrale nur begrenzt. Klaus König, von dem eine ganze Reihe wichtiger Beiträge zur Regierungszentralenforschung stammt, verweist auf die strukturelle Vielfalt und funktionalen Gemeinsamkeiten von Regierungszentralen in westlichen Demokratien und zeigt damit Potenziale für komparative Analysen auf. Auf die sub-nationale Ebene gehen die Autoren der NRWSchool of Governance ein, wobei der Beitrag von Martin Florack hervorzuheben ist, der die Rolle der Düsseldorfer Staatskanzlei am Fallbeispiel des Gesetzgebungsverfahrens zum „Hochschulfreiheitsgesetz" analysiert. Insgesamt verdeutlicht die von Stefan Bröchler und Julia von Blumenthal vorgelegte Bestandsaufnahme, dass die Regierungszentralenforschung ein lohnenswertes Gebiet darstellt; allerdings hat sie - ähnlich wie die Führungsforschung - mit der Schwierigkeit zu kämpfen, dass empirische Daten nicht uneingeschränkt erhoben werden können.

Manuela Glaab

\title{
Lesenswerte Bestätigung der Grenzen strategischer Planung in den Bundesländern
}

Schwickert, Dominic: Strategieberatung im Zentrum der Macht. Strategische Planer in deutschen Regierungszentralen, VS Verlag für Sozialwissenschaften, Wiesbaden 2011, 230 Seiten, € 34,95 .

Strategische Planung ist im politischen Betrieb selbstverständlich - und schwierig. Für wie selbstverständlich ein strategisches Vorgehen gehalten wird, lässt schon das verwendete Vokabular erahnen: Themen erfordern beim politischen Personal eine „Positionierung“, um sie inhaltlich „besetzen“ zu können. „Aktionspläne“, „Handlungskonzepte“ oder „Master- 
pläne" entstehen keineswegs nur in Krisensituationen, sondern gehören zum Standardrepertoire von Entscheidern. Auf kaum einen Vorwurf dürften Politiker so allergisch reagieren wie auf den der Konzeptlosigkeit. Vermutlich gibt es im Wochenkalender jedes Berufspolitikers Termine für Strategiegespräche. Das führt im Alltag tendenziell zu einem Überangebot an Konzeptpapieren und strategischen Entwürfen.

Dieses Überangebot korreliert allerdings mit einem erheblichen Forschungsdefizit. Kaum eine empirisch untermauerte Vermutung über das, was strategische Planer anstreben und leisten, lässt sich mit den Instrumenten von Steuerungstheorie, Policy- oder Governance-Forschung aufbereiten. Das beginnt beim Begriff: Eine "politische Strategie“ zu verfolgen, bedeutet nicht nur die Vorbereitung und Begleitung (partei-)politischer Entscheidungen oder deren möglichst erfolgreiche Umsetzung und mediale Aufbereitung, sondern weist zum Beispiel in der Themenwahl über den politischen Alltag hinaus. Das führt unweigerlich zu einer analytischen Unschärfe des Begriffs, denn damit gibt es kaum ein Thema, das nicht zum Gegenstand strategischer Überlegungen erklärt werden könnte.

Dass die Wissenschaft wenig Verlässliches über politische Planungsprozesse und deren Reichweite weiß - weniger zumindest als Journalisten in Hintergrundgesprächen erfahren können -, hat im Wesentlichen forschungspraktische Gründe. Zwar weisen fast alle politischen Institutionen Abteilungen, Stäbe oder Referate auf, die als „strategisch“ etikettiert sind oder „Planung“ als Aufgabe nennen. Inwieweit diese Einheiten aber für Erfolg oder Misserfolg beziehungsweise Zieldefinition und Ergebnisse der Institutionen verantwortlich zeichnen, das heißt inwiefern sie tatsächlich steuern, ist schwer zu messen. Außerdem dringt darüber - wenig überraschend - fast nichts nach außen, denn Zurückhaltung ist ein zentraler Teil des Tätigkeitsprofils erfolgreicher Planer.

Dominic Schwickerts Studie ist deshalb in zweierlei Hinsicht wichtig und verdienstvoll. Zum einen werden die Techniken und institutionellen Einwirkungsmöglichkeiten politisch-administrativer Planer analysiert. Zum anderen aber zeigen die Ergebnisse des Buches einen eher ratlosen Autor, der die Feststellungen und Ausführungen seiner Interviewpartner kaum mit den im Theorieteil des Buches formulierten Erwartungen zusammenzubringen vermag. Seine Untersuchungsergebnisse sind ein Gewinn für jeden politisch Interessierten und die Politikwissenschaft. Sie zeigen aber auch die erheblichen Defizite der einschlägigen Theorien und lassen vermuten, dass die Kontingenz politischer Entscheidungsprozesse sich einem theoretischen Zugang weitgehend entzieht.

Die Studie profitiert von einem sehr schlanken Forschungsdesign, leidet aber wie zahlreiche andere Qualifikationsarbeiten unter zu viel methodischem Ballast (Kapitel 4) und Referenzen an einen Theoriediskurs, der Begriffe entwickelt, aber kaum noch an praktisch implementierter Politik interessiert zu sein scheint (Kapitel 2 bis 3). Der von Neugier und Sachkenntnis angeleitete Blick des Autors auf sein Thema ist, auch wenn sich das in der Gewichtung der Kapitel kaum niederschlägt, empirieorientiert. Der kenntnisreiche Theorieteil nimmt über die Hälfte des Buchs ein, liefert aber letztlich wenig mehr als eine Pflichtübung, bei der aus dem akteurzentrierten Institutionalismus zehn Leitfragen abgeleitet werden, die der Autor auch ohne theoretische Fundierung mühelos hätte formulieren können.

Gegenstand seiner empirischen Untersuchung ist die Strategieberatung in den Regierungszentralen deutscher Länder. Damit wird strategische Planung in Parteizentralen ebenso aus dem Forschungsdesign exkludiert wie die strategische Planung in Fachressorts, Organisationen oder in der personell größten politischen Planungseinheit der Bundesrepublik, 
dem Bundeskanzleramt. Doch das ist kein Nachteil. Durch den engen Untersuchungsfokus auf die tatsächlichen (oder vermuteten) Querschnittsstrategen in den Landesregierungen gelingt Schwickert eine sehr detailgesättigte Analyse der Handlungsmöglichkeiten und -instrumente. Auf der Basis von zehn leitfadengestützten Interviews werden das Selbstverständnis der Planer, die jeweiligen Organisationszuschnitte sowie ihr Instrumentenkasten anschaulich gemacht. Durch die zugesicherte Anonymisierung ihrer Einschätzungen geben die Interviewpartner einen sehr tiefen Einblick in ihre Arbeit. Gleichwohl ist zu vermuten, dass sie nicht alle Karten auf den Tisch legen, insbesondere im Hinblick auf die zu unterstellende parteipolitische Überformung ihrer Arbeit (etwa in Wahlkämpfen).

Durchweg alle Interviewpartner berichten von einem Arbeitsalltag, in dem verwaltungstechnische Routinevorgänge und behördeninterne Abstimmungen die Planung und Strategieberatung überlagern. Einem strategischen Masterplan für das Regierungshandeln während einer Wahlperiode (oder darüber hinaus) steht die von allen Akteuren beschriebene Kurzatmigkeit des politischen Betriebs entgegen. Für Strategie bleibe im medial beschleunigten Alltagsgeschäft, so der Tenor der meisten Interviewten, kaum Zeit.

Schwickert schließt auf strukturell unzureichende Rahmenbedingungen für eine strategisch ausgerichtete Regierungspolitik und stellt fest, dass strategische Beratung, um durchsetzungsfähig zu sein, nicht nur das verwaltungstechnische Handwerk beherrschen, sondern eben auch das Ohr des Regierungschefs finden muss. Für eine Ausschöpfung ihrer kreativen und intellektuellen Potenziale fehlten den Planern in der Regel Zeit und Kapazitäten. Letztlich seien sie Planer nichts anderes als privilegierte, gut informierte, aber arbeitsüberlastete Verwaltungsmitarbeiter. Den interessierten Laien überraschen diese Thesen ebenso wenig wie den Eingeweihten, dem es allerdings an Zeit mangeln dürfte, dieses lesenswerte Buch aufzuschlagen.

Danko Knothe

\section{Viele unverbundene Perspektiven auf Politik und Marketing}

Alemann, Ulrich von, Martin Morlok und Julian Krüper (Hrsg.): (Partei-)Politik im Zeichen des Marketing (Schriften zum Parteienrecht und zur Parteienforschung, Band 40), Nomos Verlagsgesellschaft, Baden-Baden 2010, 145 Seiten, € 29,-.

Der vorliegende Sammelband ist der Ergebnisbericht des gleichnamigen Symposions, das am 18. und 19. April 2008 in Düsseldorf stattfand. Ausrichter war das Institut für Deutsches und Europäisches Parteienrecht und Parteienforschung. „Heute betreten wir Neuland, denn wir nehmen eine dritte Bezugswissenschaft hinzu, die Ökonomie. Heute sind wir nicht ein bi-disziplinäres Institut, sondern wir begehen ein tri-disziplinäres Symposion“ (S. 9), schreibt Ulrich von Alemann in der Einführung. Bereits der Titel des Buches verweist auf zwei der interdisziplinären Betrachtungsweisen, über die von Alemann referiert. Zum einen wird der Kernbereich der Politikwissenschaft in Form der (Partei-)Politikforschung angesprochen. Zum anderen wird der Begriff Marketing als einer der Grundpfeiler der Wirtschaftswissenschaft in die Diskussion eingebracht. Die dritte Dimension gewinnt das Symposion durch die inhaltliche Ausrichtung des Institutes für Deutsches und Euro- 